\title{
When THE HEART KiLls THE Liver: Acute Liver Failure in Congestive Heart Failure
}

\author{
F. H. Saner ${ }^{1}$, M. Heuer ${ }^{1}$, M. Meyer ${ }^{1}$, A. Canbay ${ }^{2}$, G. C. Sotiropoulos ${ }^{1}$, A. Radtke ${ }^{1}$, J. Treckmann ${ }^{1}$, \\ S. Beckebaum ${ }^{1}$, C. Dohna-Schwake ${ }^{2}$, S. W. Oldedamink ${ }^{3,4}$, A. Paul ${ }^{1}$ \\ ${ }^{1}$ Department of General-, Visceral- and Transplant Surgery, University Hospital Essen, Germany, \\ ${ }^{2}$ Department of Pediatric Medicine, University Hospital Essen, Germany, \\ ${ }^{3}$ Department of Surgery, University of Maastricht, Netherlands, \\ ${ }^{4}$ Department of Surgery, University College London Hospital, UCL, UK
}

\begin{abstract}
Congestive heart failure as a cause of acute liver failure is rarely documented with only a few cases.

Although the pathophysiology is poorly understood, there is rising evidence, that low cardiac output with consecutive reduction in hepatic blood flow is a main causing factor, rather than hypotension. In the setting of acute liver failure due to congestive heart failure, clinical signs of the latter can be absent, which requires an appropriate diagnostic approach.

As a reference center for acute liver failure and liver transplantation we recorded from May 2003 to December 2007202 admissions with the primary diagnoses acute liver failure. 13/202 was due to congestive heart failure, which was associated with a mortality rate of $54 \%$. Leading cause of death was the underlying heart failure. Asparagine transaminase (AST), bilirubin, and international normalized ratio (INR) did not differ significantly in surviving and deceased patients at admission. Despite both groups had signs of cardiogenic shock, the cardiac index (CI) was significantly higher in the survival group on admission as compared with non-survivors $\left(2.1 \mathrm{~L} / \mathrm{min} / \mathrm{m}^{2}\right.$ vs. 1.6 $\left.\mathrm{L} / \mathrm{min} / \mathrm{m}^{2}, \mathrm{p}=0.04\right)$. Central venous - and pulmonary wedge pressure did not differ significantly. Remarkable improvement of liver function was recorded in the group, who recovered from cardiogenic shock.

In conclusion, patients with acute liver failure require an appropriate diagnostic approach. Congestive heart failure should always be considered as a possible cause of acute liver failure.
\end{abstract}

Key words: Acute liver failure, congestive heart failure, Doppler ultrasound

\section{INTRODUCTION}

Acute liver failure (ALF) is defined as an abrupt onset of jaundice, hepatic encephalopathy, and coagulopathy in the absent of pre-existing liver disease [11]. Cardiomyopathy as the underlying cause of ALF is rare and only a few case reports are documented in the literature $[5,7,8,18]$. Cardiac decompensation can initially be undetected, and the usual signs of con- gestive heart failure may be absent [5, 18]. Both, chronic and acute congestive heart failure can lead to hepatic dysfunction [10,17]. Although there is no classic pattern of abnormalities, a cholestatic biochemical profile is common, with a mild elevation in total bilirubin (usually $3 \mathrm{~g} / \mathrm{dL}$ ), a mild elevation in alkaline phosphatase and only occasional elevations in transaminases. Another common observation is an increase in INR. The presumed causes of hepatic dysfunction in congestive heart failure are hepatic congestion from venous outflow obstruction and resulting hypertension and decreased oxygen delivery from an impaired cardiac output [10]. The latter has been hypothesised to result from the fairly fixed portion of the cardiac output that supplies the hepatic bed (about 20\%). Once the decrease in hepatic oxygen delivery crosses a critical threshold, a cascade of events is initiated that ultimately results in hemorrhagic centrilobular necrosis $[2,10]$.

During a five year period we identified 13 patients with ALF due to congestive heart failure, which was diagnosed by right heart catheterisation, transthoracic echocardiography and laboratory data.

The aim of our study was to determine the clinical course and laboratory features in patients with ALF induced by congestive heart failure.

\section{Patients And Methods}

The study was conducted in accordance with the Declaration of Helsinki.

Between May 2003 and December 2007 we recorded 202 admissions at our Intensive Care Unit (ICU) with the primary diagnoses ALF. 95 of these patients had signs of an acute decompensation of chronic liver disease. The remaining patients fulfilled the criteria of ALF according to O'Grady et al. [11]. 13 of these 107 patients $(10.3 \%)$ suffered from ALF caused by congestive heart failure (see Fig. 1). Interestingly, 7 of the 13 patients were referred from cardiology departments. Although, patients had a well known history of chronic heart deficiency, all referring centers excluded any association between cardiac failure and ALF due to absence of clinical signs of cardiac 


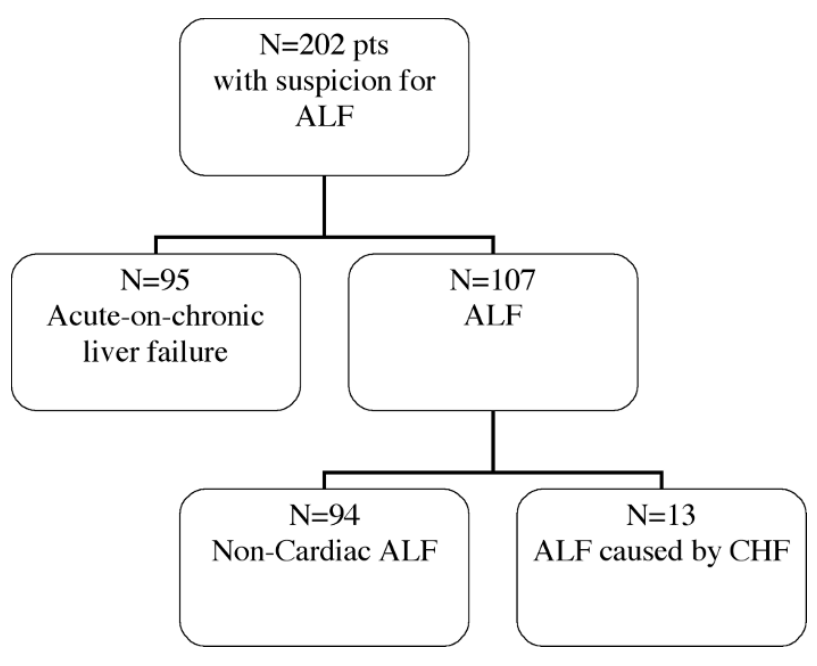

Fig. 1. Admission at Intensive Care Unit (ICU) with the primary diagnoses acute liver failure in the time period 5/200312/2007. Pts: patients; ALF: acute liver failure, CHF: congestive heart failure.

decompensation and unchanged transthoracic echocardiography as compared with earlier examinations. However, hemodynamic evaluations with right heart catheter were not performed in these centers. The remaining 6 patients were referred from general medical wards.

\section{Standard Procedure for Patients with Acute LIVER FAILURE}

Our workup in all patients, who were admitted with the suspicion of ALF consists of an ultrasound Doppler, particular of the liver, to exclude an acute Budd-Chiari syndrome. Blood flow measurements were performed using sonographic equipment with color Doppler capability (Siemens Sonoline, Erlangen, Germany) and a $3.5 \mathrm{MHz}$ probe to determine tracing in the portal vein (PV), the hepatic artery (HA) and the hepatic vein (HV). Moreover, special laboratory investigation for all other possible diagnoses which are associated with an ALF, e.g. viral hepatitis (A and B), autoimmune hepatitis, M. Wilson, drug-related toxicity (particular acetaminophen), HELLP -syndrome were performed. Laboratory routine work-up was performed daily and included at least total bilirubin, AST, alanine transaminase (ALT), and INR.

\section{Assessment of Heart Failure}

In cases, where the cause of ALF remained unknown the patients were scheduled for a right heart catheterisation to exclude cardiac failure.

Normal values for the pulmonary artery catheterization assessment were defined according to Shoemaker [14]:

Cardiac Index (CI): $2.8 \mathrm{~L} / \mathrm{min} / \mathrm{m}^{2}$, pulmonary capillary wedge pressure

(PCWP): 12-15 mmHg, central venous pressure $(C V P): 5-10 \mathrm{mmHg}$
Systemic vascular resistance (SVR):

900-1500 dyn* sec* $^{*} \mathrm{~cm}^{-5}$,

pulmonary vascular resistance (PVR):

$90-180 \mathrm{dyn}^{*} \sec ^{*} \mathrm{~cm}^{-5}$

Mean arterial pressure (MAP), mean pulmonary arterial pressure (MPAP), CVP, and PCWP were recorded using standard disposable pressure transducers (Medex Medical, Klein-Winternheim, Germany), together with leads II and V5 of the electrocardiogram for the detection of heart rate (Sirecust 1281 Siemens, Germany). Cardiac output was measured by a thermodilution method, using the Stewart-Hamilton equation (18). The CI was calculated by dividing the cardiac output by the Body surface area (BSA). Transthoracic echocardiography was used for global assessment of the heart, estimating of the ejection fraction and contractility of the right and left ventricle.

\section{STATISTICAL ANALYSIS}

The categorical variables were analyzed by Chi-squared test with Yates correction; whereas continuous variables were analyzed by one-way analysis of variance and post hoc Scheffe test when normal distribution was given. Non-normally distributed continuous variables were analyzed by Kruskal-Wallis one-way analysis of variance on ranks. $\mathrm{P}<0.05$ was considered significant.

\section{RESULTS}

We identified 13 patients (male: 8, female: 5) during the time period of May 2003 to December 2007 with acute liver failure caused by cardiac failure (Fig. 1). The mean age of the patients was $48.4 \pm 17.4$ years. All patients suffered from cardiogenic shock at admission.

\section{Causes of Heart Failure}

Six patients suffered from dilatative cardiomyopathy (ejection fraction $\leq 20 \%$ ) with acute decompensation. Two patients developed pericardial tamponade following cardiac surgery. One patient suffered from pulmonary embolism and consecutive right heart failure, one patient experienced aortic valve stenosis $\mathrm{III}^{\circ}$ (aortic root $0.4 \mathrm{~cm}^{2}$ ) with secondary pulmonary hypertension and right heart failure. Two patients were admitted with a myocardial infarction, and one patient with a known primary pulmonary hypertension had an acute right heart failure due to tachyarrhythmia absoluta.

\section{Outcome}

The overall hospital mortality rate was $54 \%$ (7 out of 13). 7 patients died within 4 days due to heart failure and were not accompanied with liver failure.

The remaining 6 patients recovered from cardiogenic shock and improved in their clinical course. The patient with the aortic valve stenosis underwent a successful valve replacement and recovered. The patient with the pulmonary embolism was treated with fibrinolyses and recovered. All surviving patients were readmitted to the referring hospital with remarkable improved cardiac - and liver function. 


\section{LABORATORY VALUES}

AST, ALT, bilirubin, and INR did not differ statistically on admission between patients, who survived or deceased. AST was significantly lower in the surviving group on day 7 as compared with all patients at admission $(1043 \pm 1013 \mathrm{U} / \mathrm{L}$ vs $4029 \pm 3514 \mathrm{U} / \mathrm{L})$. Although, bilirubin, ALT decreased and INR increased, these apparent differences did not reach statistically significance (see Table 1 and Fig. 2)

Table 1. Laboratory and hemodynamic values at time of transfer to our department and at day 7 of the survived patients.

\begin{tabular}{lcc}
\hline Value & Admission & Day 7 \\
\hline AST (U/L) & $4029 \pm 3514$ & $1043 \pm 1013^{*}$ \\
ALT (U/L) & $2685 \pm 2088$ & $1193 \pm 1068$ \\
Bilirubin (U/L) & $4.3 \pm 3.1$ & $2.2 \pm 1.3$ \\
INR & $2.4 \pm 1.3$ & $1.7 \pm 0.5$ \\
HR $(1 / \mathrm{min})$ & $105 \pm 26$ & $115 \pm 28$ \\
MAP (mmHg) & $71 \pm 15$ & $73 \pm 9$ \\
CVP (mmHg) & $16 \pm 5$ & $11 \pm 2 *$ \\
CI $\left(\mathrm{L}^{*} \mathrm{~min}^{*} \mathrm{~m}^{2}\right)$ & $1.9 \pm 0.9$ & $3 \pm 1 *$ \\
MPAP $\left(\mathrm{mmHg}^{2}\right)$ & $40 \pm 19$ & $27 \pm 7$ \\
PCWP (mmHg) & $20 \pm 10$ & $15 \pm 5$ \\
SVR (dyn*sec*cm-5) & $1713 \pm 525$ & $922 \pm 236$ \\
PVR (dyn*sec*cm-5) & $327 \pm 146$ & $202 \pm 49$ \\
Noradrenaline $(\mu \mathrm{g} / \mathrm{kg} / \mathrm{min})$ & $0.9 \pm 0.6$ & $0.2 \pm 0.1 *$ \\
\hline
\end{tabular}

$* \mathrm{P}<0.05$

\section{HEMODYNAMICS}

All patients suffered from cardiogenic shock (CI $=1.9$ $\mathrm{L} / \mathrm{min} / \mathrm{m}^{2}$ ). However, patients who survived had significant higher $\mathrm{CI}$ as compared with non-survivors $\left(2.1 \pm 0.2 \mathrm{~L} / \mathrm{min} / \mathrm{m}^{2}\right.$ vs. $\left.1.6 \pm 0.5 \mathrm{~L} / \mathrm{min} / \mathrm{m}^{2}\right)$. There were no significant differences in other cardiovascular parameters between both groups on admission [CVP $(17 \pm 4 \mathrm{mmHg}$ in survival group vs, $15 \pm 6 \mathrm{mmHg}$ non-survival), PCWP (20 $\pm 6 \mathrm{mmHg}$ survival group, $20 \pm 12 \mathrm{mmHg}$ non-survival group), and MPAP (32 \pm $10 \mathrm{mmHg}$ survival group vs. $46 \pm 21 \mathrm{mmHg}$ non-survival group)].

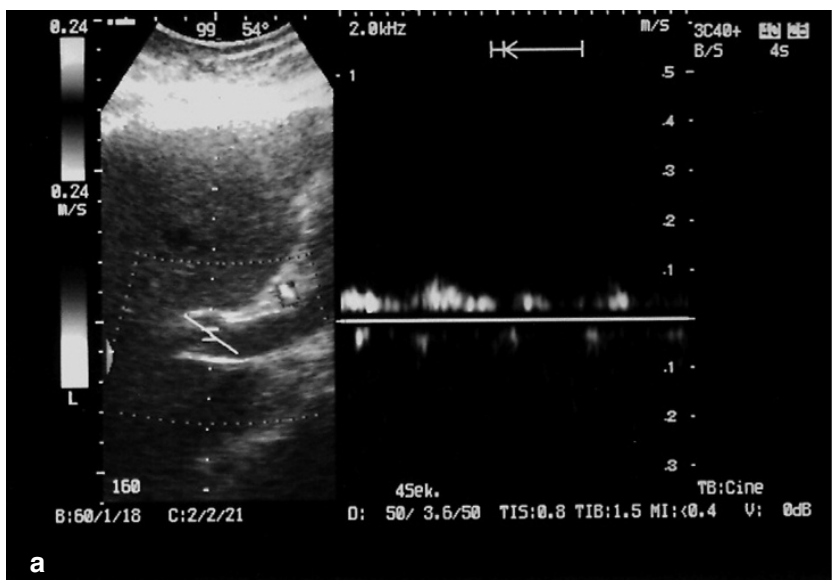

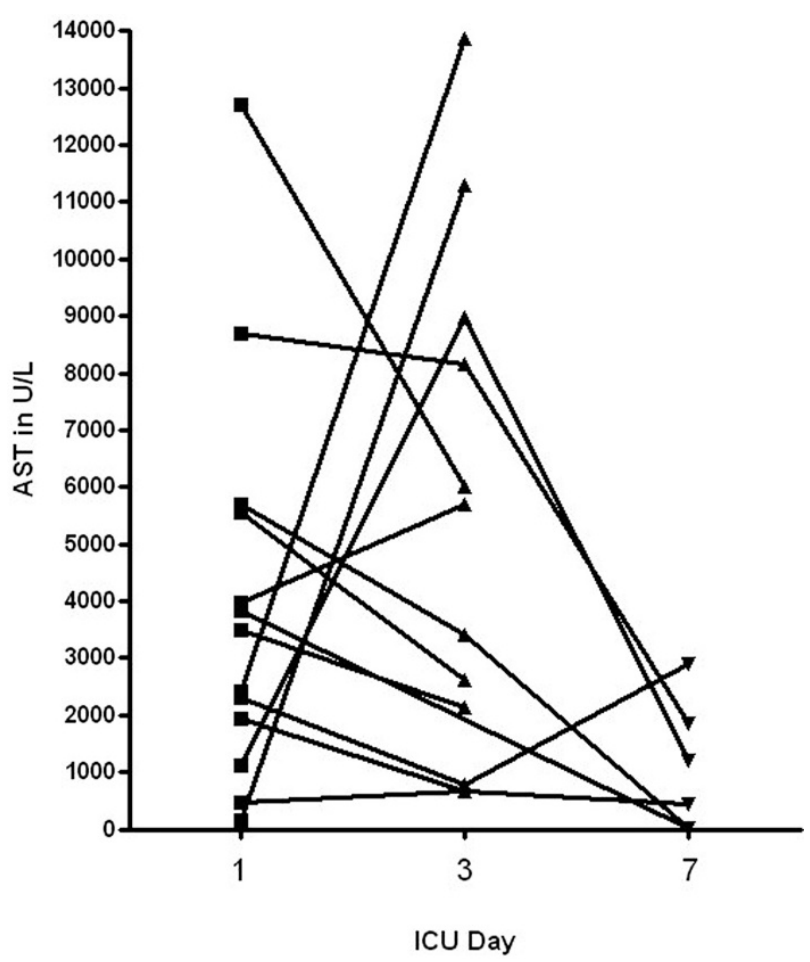

Fig. 2. Asparagine transaminase (AST) course of all patients during 1 week after admission. On day 7 only 6 of 13 patients were alive and showed a significant AST decrease as compared with baseline on admission.

Echocardiogram was performed in all patients that revealed global hypokinesis with estimated ejection fraction $\leq 20 \%$.

Dobutamine as inotropic support was infused in 3 patients (dose $2-8 \mu \mathrm{g} / \mathrm{kg} / \mathrm{min}$ ), milrinone was used for 2 patients (dose $0.5-3 \mu \mathrm{g} / \mathrm{kg} / \mathrm{min}$ ), or adrenaline (8 patients, dose $\leq 0.1 \mu \mathrm{g} / \mathrm{kg} / \mathrm{min})$. Noradrenaline as a vasopressor was used in all patients to maintain a MAP of $70 \mathrm{mmHg}$.

Patients who survived showed a significant increase of CI at day 7 compared with all patients at admission. CVP and vasopressor support (noradrenalin) was significantly reduced at day 7 in the survival group as compared with baseline (see Table 1).

Fig. 3. Portal vein tracing showed a partial reversed flow on admission (a). After cardiac recovery the flow pattern remained pulsatile, but hepatopedal (b).

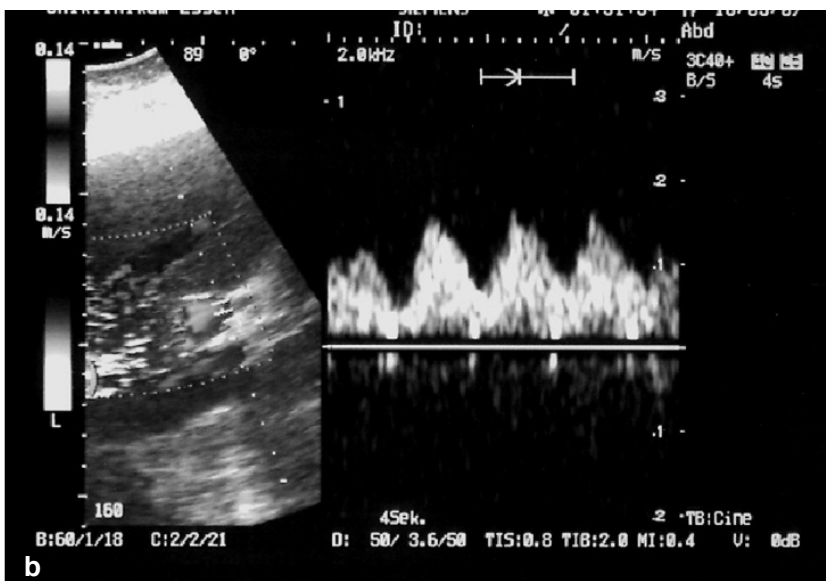



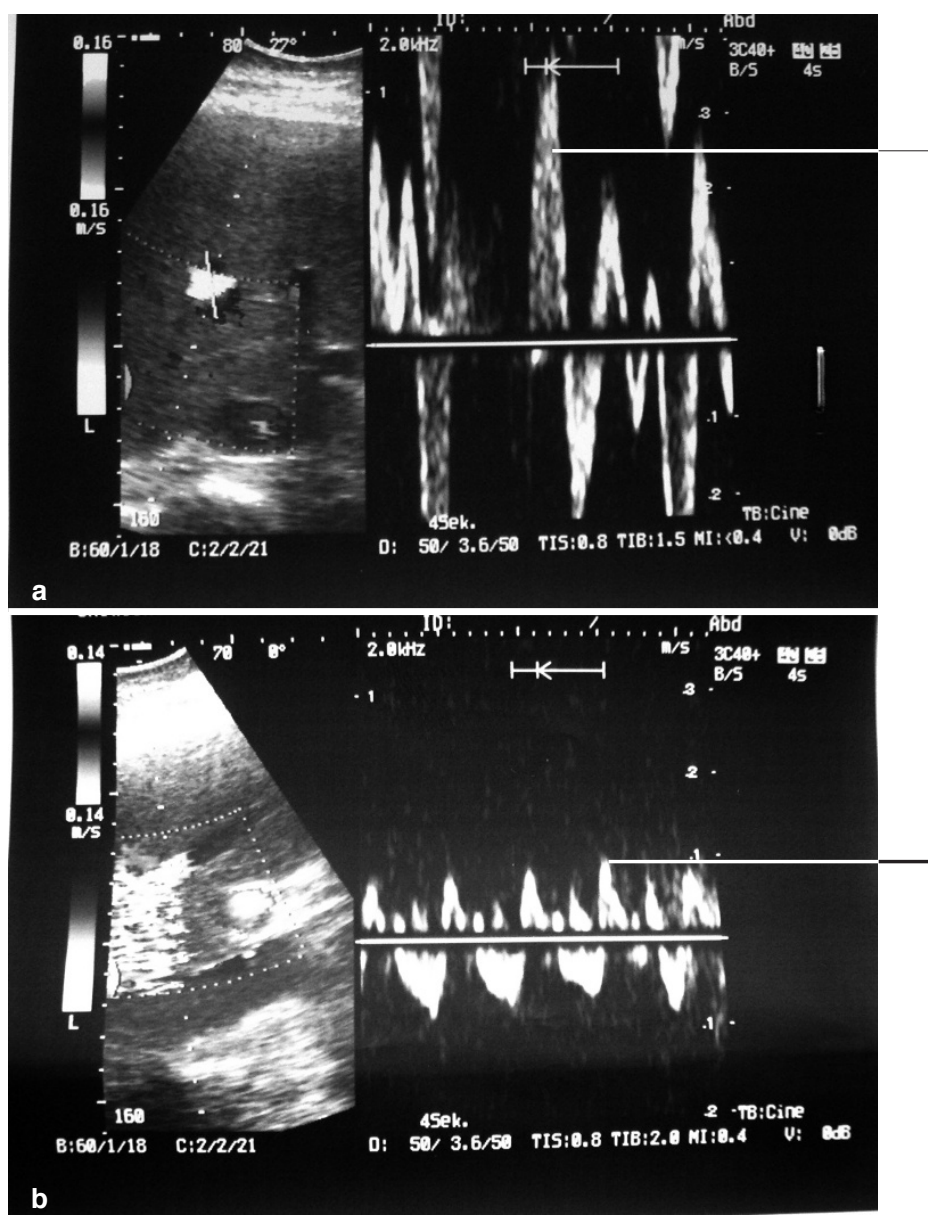

High backflow into the liver due to cardiogenic shock

Remarkable decreased backflow after cardiac recovery

Fig. 4. Doppler wave form of the hepatic vein (middle). The trace showed a triphasic wave with an increase of the retrograde phase in the hepatic vein (a). After cardiac recovery the hepatic vein tracing showed nearly normal pattern, with remarkable decrease of the retrograde flow (b).

\section{AbDominal Ultrasound AND Doppler INVESTIGATION}

In all patients the doppler tracing documented severe impairment of portal vein flow with partial reversed flow without signs of a portal vein thrombosis (see Fig. 3). A repeated ultrasound in the survival group on day 5 showed a remarkable improved portal vein tracing with pulsatile, but hepatopedal flow pattern.

The hepatic vein preserved its triphasic pattern, however with a remarkable increased retrograde flow (see Fig. 4). After cardiac recovery the retrograde flow decreased. The hepatic artery showed a regular flow tracing despite a high dose of vasopressor treatment.

\section{Discussion}

In our study we identified 13 patients with ALF caused by congestive heart failure. All patients suffered from cardiogenic shock, however patients that survived had significant higher CI on admission as compared with non-survivors. The overall hospital mortality was 54\%. Patients who survived had a regular CI on day 7, which was associated with improved liver function. Ultrasound Doppler images on admission showed pathological traces of portal- and hepatic vein, which were reversed after cardiac recovery.
Heart failure is a most often accompanied by of elevated plasma concentrations of liver enzymes [10, 17]. Only Kubo et al. [9] reported a slight AST elevation $(65 \pm 82 \mathrm{U} / \mathrm{L})$ in patients with a CI $\leq 1.5$ $\mathrm{L} / \mathrm{min} / \mathrm{m}^{2}$. However, most other reports (Wiesen et al. [18]; Fussell et al. [4]) support the findings of our present study, where AST concentrations were shown to be elevated more than 2000 -fold, despite a mean CI of $1.9 \mathrm{~L} / \mathrm{min} / \mathrm{m}^{2}$. The main difference of Kubo's report and the other studies are that Kubo described the incidence and severity of liver function abnormalities in patients with congestive heart failure, but in absence of acute decompensation. Despite a very low CI in his study, which did occur not abruptly, compensatory mechanisms may react (additional liver veins draining from diaphragmal veins or vena gastrica sinistra), if there is time enough to create this bypass.

Fulminant hepatic failure caused by congestive heart failure has rarely been documented; furthermore the exact pathophysiology remains unclear. Seeto et al. [13] stated that systemic hypotension or shock alone did not lead to liver failure in any patient. The majority of patients in their study with liver failure had severe underlying cardiac disease that had often led to passive congestion of the liver. They conclude that right-sided heart failure resulting in hepatic venous congestion, may predispose the liver to hepatic injury induced by a hypotensive event. The most presumed causes of car- 
diac induced ALF are hepatic congestion from venous hypertension and decreased oxygen delivery (DO) from a decreased CI [17]. Once DO underwent a critical threshold, hepatic hypoxia initiates a process that results in hemorrhage centrilobular necrosis [2]. Venous hypertension results in periventricular sinusoidal congestion, endothelial injury, replacement of hepatocytes with erythrocytes, and ultimatively, centrilobular necrosis $[2,6]$. In our study patients fulfilled all criteria for cardiogenic shock with elevated CVP. Patients who survived and recovered from cardiogenic shock remained a high CVP, but showed an improvement in liver function. This led us to the conclusion that a low CI has more impact on the liver congestion than a high CVP. Indeed, a low CI induces a high CVP which ultimately may cause liver congestion. However, an association of liver failure with high CVP (> $10 \mathrm{mmHg}$ ) as a sign of volume overload but regular CI has never been reported.

The Doppler ultrasound pattern of reversed pulsatile flow (RPF) of the PV is strongly associated with high right atrial pressure $(>20 \mathrm{mmHg})[1,12]$. Tricuspid regurgitation is considered to be the main cause of RPF in patients with congestive heart failure, but the precise pathophysiological mechanism of the portal veins RPF remains unsolved. In our study the PV flow was only partially reversed, but with very severe impaired hepatopedal flow. After improvement of cardiac function the hepatopedal flow improved but remained pulsatile. A very similar RPV was reported in patients with a Budd-Chiari syndrome [16]. This syndrome leads to occlusion of the hepatic vein and/or inferior vena cava and therefore resembles the situation of elevated right atrial pressure. The venous outflow obstruction of the liver leads to increased portal vein and hepatic sinusoid pressures as the blood flow stagnates with total or partial RPV flow [3].

Hepatic vein flow in healthy volunteers consists of two forward flows toward the heart; one systole, the other one in diastole, and one retrograde flow toward the liver during atrial contraction [15]. In patients with atrial fibrillation, and more pronounced in patients with congestive heart failure a systolic backward flow toward the liver is reported [19]. In our study we found a major backflow toward the liver at both cycle systolic and diastolic. After cardiac recovery the hepatic vein flow improved and was mainly directed out of the liver.

As any observational cohort study, our report has some limitations. First of all we performed a retrospective study were the findings of such an investigation carry less persuasive force than those of a prospective study. In order of our limited sample size we were not able to abbreviate special recommendation for treatment for these patients. Moreover we were not able to make any conclusions about the epidemiology of ALF caused by cardiac failure. However, what we learned form our data is that every patient with unknown cause of ALF should be evaluated with right heart catheter to exclude cardiac induced liver failure. The only curative treatment option for these patients is to improve cardiac function.

In conclusion, ALF induced by heart failure is rare and carries a high mortality rate. In case of unknown cause of ALF, despite a meticulous workup, cardiac failure should always be considered. Right heart catheter evaluation for exact assessment of CI, MPAP, and CVP is mandatory for these patients.

The authors declare that they have no competing interests.

\section{REFERENCES}

1. Abu-Yousef M. M., Milam S. G. and Farner R. M. Pulsatile portal vein flow: a sign of tricuspid regurgitation on duplex Doppler sonography. AJR Am J Roentgenol 1990; 155 (4): 785-8.

2. Arcidi J. M., Jr., Moore G. W. and Hutchins G. M. Hepatic morphology in cardiac dysfunction: a clinicopathologic study of 1000 subjects at autopsy. Am J Pathol 1981; 104 (2): 159-66.

3. Boozari B., Bahr M. J., Kubicka S., Klempnauer J., Manns M. P. and Gebel M. Ultrasonography in patients with Budd-Chiari syndrome: diagnostic signs and prognostic implications. J Hepatol 2008; 49 (4): 572-80.

4. Fussell K. M., Awad J. A. and Ware L. B. Case of fulminant hepatic failure due to unrecognized peripartum cardiomyopathy. Crit Care Med 2005; 33 (4): 891-3.

5. Hoffman B. J., Pate M. B., Marsh W. H. and Lee W. M. Cardiomyopathy unrecognized as a cause of hepatic failure. J Clin Gastroenterol 1990; 12 (3): 306-9.

6. Kanel G. C., Ucci A. A., Kaplan M. M. and Wolfe H. J. A distinctive perivenular hepatic lesion associated with heart failure. Am J Clin Pathol 1980; 73 (2): 235-9.

7. Kaymakcalan H., Dourdourekas D., Szanto P. B. and Steigmann F. Congestive heart failure as cause of fulminant hepatic failure. Am J Med 1978; 65 (2): 384-8.

8. Kisloff B. and Schaffer G. Fulminant hepatic failure secondary to congestive heart failure. Am J Dig Dis 1976; 21 (10): 895-900.

9. Kubo S. H., Walter B. A., John D. H., Clark M. and Cody R. J. Liver function abnormalities in chronic heart failure. Influence of systemic hemodynamics. Arch Intern Med 1987; 147 (7): 1227-30.

10. Naschitz J. E., Slobodin G., Lewis R. J., Zuckerman E. and Yeshurun D. Heart diseases affecting the liver and liver diseases affecting the heart. Am Heart J 2000; 140 (1): $111-20$

11. O'Grady J. G., Schalm S. W. and Williams R. Acute liver failure: redefining the syndromes. Lancet 1993; 342 (8866): 273-5.

12. Rengo C., Brevetti G., Sorrentino G., D'Amato T., Imparato M., Vitale D. F., Acanfora D. and Rengo F. Portal vein pulsatility ratio provides a measure of right heart function in chronic heart failure. Ultrasound Med Biol 1998; 24 (3): 327-32.

13. Seeto R. K., Fenn B. and Rockey D. C. Ischemic hepatitis: clinical presentation and pathogenesis. Am J Med 2000; 109 (2): 109-13.

14. Shoemaker W. C. Relation of oxygen transport patterns to the pathophysiology and therapy of shock states. Intensive Care Med 1987; 13 (4): 230-43.

15. Teichgraber U. K., Gebel M., Benter T. and Manns M. P. [Duplex ultrasound characterization of hepatic vein blood flow in healthy probands]. Ultraschall Med 1997; 18 (6): 267-71.

16. Wachsberg R. H., Needleman L. and Wilson D. J. Portal vein pulsatility in normal and cirrhotic adults without cardiac disease. J Clin Ultrasound 1995; 23 (1): 3-15.

17. Ware A. J. The liver when the heart fails. Gastroenterology 1978; 74 (3): 627-8.

18. Wiesen S., Reddy K. R., Jeffers L. J. and Schiff E. R. Fulminant hepatic failure secondary to previously unrecognized cardiomyopathy. Dig Dis 1995; 13 (3): 199-204. 
19. Yoshida K., Yoshikawa J., Kato H., Yanagihara K., Okumachi F., Koizumi K., Shiratori K., Asaka T., Suzuki K. and Inanami H. [Tricuspid regurgitation evaluated by Doppler hepatic vein flow patterns]. J Cardiogr 1985; 15 (4): $1157-69$.

Received: July 28, 2009 / Accepted: August 14, 2009
Address for correspondence:

Priv. - Doz. Dr. med. F. Saner, DEAA

University Essen

Department of General-, Visceral- and Transplant Surgery

Hufelandstr. 55

45122 Essen

Germany

Tel.: $\quad+49-201-723-1177$

Fax.: +49-201-723-1196

E-mail: fuat.saner@uni-due.de 